Table 1 PTU-019 Severity and duration

\begin{tabular}{lcccl}
\hline & & $\begin{array}{l}\text { duration 1st and } \\
\text { 2nd quartile } \\
\text { ( } 10 \text { days })\end{array}$ & $\begin{array}{l}\text { duration 3rd } \\
\text { and 4th quartile } \\
\text { (> 10 days) }\end{array}$ & p Value \\
\hline SIRS present - $\mathrm{n}(\%)$ & 47 & $8(33)$ & $10(43)$ & 0.47 \\
WBC, $\times 10^{9} / \mathrm{I}$ - median (IOR) & 47 & $12.9(8.8-19)$ & $10.4(7.8-17)$ & 0.41 \\
albumin, g/l - median (IOR) & 44 & $33.5(27-38)$ & $33(31-37)$ & 0.66 \\
creatinine, mmol/I - median (IQR) & 47 & $73(55-90)$ & $79(61-126)$ & 0.43 \\
CRP, mg/l - median (IOR) & 46 & $140(24-160)$ & $102(43-160)$ & 0.78 \\
\hline
\end{tabular}

faecal $C$ difficile culture from an ongoing study of CDI at our institution. Data were analysed using STATA10.

Results Seventy-six patients met the case definition, of which $60(79 \%)$ had notes available. Poor documentation in 6 $(10 \%)$ and discharge with ongoing diarrhoea in $7(12 \%)$ made it impossible to determine the duration of diarrhoea. In the remaining 47 patients $(78 \%)$, the median duration of diarrhoea was 10 days (IOR, 6-20.5 days). Ten patients (21\%) had symptoms for 3 weeks or more. Duration was independent of age $(p=0.78)$ and of clinical and laboratory markers of severity (table 1). Five patients (23\%) with diarrhoea persisting beyond the median were culture negative compared with $3(13 \%)$ of those with shorter duration although this difference was not statistically significant $(\mathrm{p}=0.3)$.

Conclusion In this study, persistence of diarrhoea was common, not limited to the elderly, and often prolonged. This places a significant burden on hospital resources. The origin of the problem is unclear but continued severe inflammation seems unlikely given the distribution of our markers of severity across the two groups. Further, false positive EIA results are well recognised; a total of $15(25 \%)$ of the 60 patients in the study were EIA positive but culture negative. This, together with the difficulty distinguishing colonisation from infection, raises the possibility that other causes of diarrhoea may have been under-diagnosed.

Competing interests None.

Keywords clostridium difficile, infectious diarrhoea.

\title{
REFERENCE
}

1. Parks T, Wallis S, Wilson J et al. Continuing diarrhoea after ten days of oral metronidazole or oral vancomycin for presumed, hospital-acquired Clostridium difficile colitis in elderly hospital patients. J Hosp Infect 2010;74:403-5.

\section{PTU-019 PERSISTENT DIARRHOEA AFTER PRESUMED CLOSTRIDIUM DIFFICILE INFECTION AT OXFORD RADCLIFFE HOSPITALS NHS TRUST: AN UNRECOGNISED ENTITY}

doi:10.1136/gut.2011.239301.147

T Parks,,${ }^{12^{*}}$ T Chapman, ${ }^{2}$ E Culver, ${ }^{2,3}$ M Scarborough ${ }^{2,3}{ }^{1}$ Clinical Academic Graduate School, University of Oxford, Oxford, UK; ${ }^{2}$ Oxford Radcliffe Hospitals NHS Trust, Oxford, UK; ${ }^{3}$ Nuffield Department of Medicine, University of Oxford, Oxford, UK

Introduction Patients presumed to have Clostridium difficile infection (CDI) often suffer from persisting diarrhoea despite appropriate antibiotic chemotherapy. This phenomenon may occur in up to $50 \%$ of patients admitted to Medicine for the Elderly. ${ }^{1}$ We set out to investigate the frequency of prolonged diarrhoea locally and to identify potential associations with age and markers of disease severity.

Methods We retrospectively reviewed the medical notes, bedside charts and laboratory results of all adult inpatients with CDI over a three month period. Our predetermined case definition was a clinical diagnosis of CDI (at the time of the illness) plus documented diarrhoea and a positive faecal toxin enzyme immunoassay (EIA). In addition, we obtained the results of 